\title{
Unbalance of intestinal microbiota in atopic children
}

\author{
Marco Candela ${ }^{1 *}$, Simone Rampelli ${ }^{1}$, Silvia Turroni ${ }^{1}$, Marco Severgnini ${ }^{2}$, Clarissa Consolandi ${ }^{2}$, Gianluca De Bellis ${ }^{2}$, \\ Riccardo Masetti ${ }^{3}$, Giampaolo Ricci ${ }^{4}$, Andrea Pession ${ }^{3}$ and Patrizia Brigidi ${ }^{1}$
}

\begin{abstract}
Background: Playing a strategic role in the host immune function, the intestinal microbiota has been recently hypothesized to be involved in the etiology of atopy. In order to investigate the gastrointestinal microbial ecology of atopic disease, here we performed a pilot comparative molecular analysis of the faecal microbiota in atopic children and healthy controls.

Results: Nineteen atopic children and 12 healthy controls aged 4-14 years were enrolled. Stools were collected and the faecal microbiota was characterized by means of the already developed phylogenetic microarray platform, HTF-Microbi.Array, and quantitative PCR. The intestinal microbiota of atopic children showed a significant depletion in members of the Clostridium cluster IV, Faecalibacterium prausnitzii, Akkermansia muciniphila and a corresponding increase of the relative abundance of Enterobacteriaceae.

Conclusion: Depleted in key immunomodulatory symbionts, the atopy-associated microbiota can represent an inflammogenic microbial consortium which can contribute to the severity of the disease. Our data open the way to the therapeutic manipulation of the intestinal microbiota in the treatment of atopy by means of pharmaceutical probiotics.
\end{abstract}

\section{Background}

Atopic diseases are chronic inflammatory disorders caused by an aberrant T-helper (Th2)-type immune response against common and innocuous environmental antigens [1]. The elaboration of cytokines, such as interleukin (IL)-4, IL-13 and IL-5, can contribute to disease induction [2]. During the past decades the prevalence of atopic diseases among children in the western world has dramatically increased [2]. Too fast for any possible shift in genetic constitution, environmental changes associated with the western lifestyle are believed to be involved $[3,4]$. The human intestinal microbial community has been indicated as a key factor to interpret the impact of the western lifestyle on the etiology of atopic diseases [3-7]. The intestinal microbiota is extremely plastic in response to diet and environmental factors and, at the same time, governs many aspects of the immune function throughout the body [8]. Thus, the

\footnotetext{
*Correspondence: marco.candela@unibo.it

'Department of Pharmaceutical Sciences, University of Bologna, Via Belmeloro 6, Bologna 40126, Italy

Full list of author information is available at the end of the article
}

hypothesis that specific western lifestyle-driven dysbioses of the human intestinal microbiota are involved in the bloom of atopy in western children has been advanced.

The human intestinal microbiota consists of a total amount of $10^{14}$ microbial cells differentiated in more than 500 different species [9]. Showing a tremendous metabolic potential, this versatile microbial "organ" exerts a role of primary importance for our metabolism. Recently, the strategic role of the intestinal microbiota in the development, education and functionality of the human innate and adaptive immune system has been recognized [7,10]. According to Gaboriau-Routhiau et al. [11], specific members of the intestinal microbial community exert an active role in the modulation of a striking range of $\mathrm{T}$ cell functions, such as Th17, Th1, Th2 and regulatory cell phenotype ( $\mathrm{T}$ regs). Having a profound impact on the overall human immune status, perturbations of the intestinal microbiota have been implicated in the development and progression of inflammatory diseases, such as inflammatory bowel diseases (IBD), autoimmune disorders, allergy and type II diabetes $[12,13]$. 
On the basis of the perceived importance of the intestinal microbiota in the education of the human immune system to tolerance [5], culture-independent perspective studies have been carried out to determine whether specific microbiota dysbioses in the early life could affect the subsequent manifestation and sensitization of atopic diseases. In the Lifestyle and Genetic Constitution (KOALA) Birth Cohort Study - an extensive epidemiological study with involved 957 infants from Netherlands aged 1 month - the presence of Escherichia coli and Clostridium difficile in stools has been associated with a higher risk to develop eczema [14]. Even if the healthpromoting microbiota components Bifidobacterium and Lactobacillus have been suggested as possible protective factors against the risk to develop atopy $[15,16]$, no differences in the prevalence of these probiotic genera between infants with and without allergic disorders have been detected $[3,14,17,18]$. More recently, two perspective surveys of the intestinal microbiota in Danish and Swedish infants have been carried out with a longitudinal approach, sampling the faecal microbiota at different time points during the first year of life $[19,20]$. Based on denaturing gradient gel electrophoresis (DGGE) and $16 \mathrm{~S}$ rDNA 454-pyrosequencing, respectively, these robust and extensive studies proved that the low bacterial diversity in the early life, rather than the prevalence of a specific bacterial taxon, is associated with an increased risk of subsequent atopic disease, reinforcing the "old friend hypothesis" [21]. According to this theory, the western lifestyle caused the disappearance of key bacterial groups from the intestinal microbiota, which are essential to prime the physiology of our immune system. The lack of these "old friends" during the perinatal period led to an immune system incline to inappropriate activation, which is a characteristic of the emerging chronic inflammatory diseases in the western world.

Even if the role of the intestinal microbiota in the predisposition to develop atopy in infancy has been accepted, to our knowledge, only few case-control culture-independent studies of the gastrointestinal microbial ecology in atopic diseases have been carried out. However, by modulating the immune status throughout the body [8], an inflammogenic gut microbial community in atopic subjects could significantly contribute to the severity of the disease. In this perspective we performed a pilot case-control study of the atopy-associated dysbiosis of the intestinal microbiota in atopic children. Since from birth to weaning the infant intestinal microbiota is an extremely dynamic entity, which continuously fluctuates in response to factors of environmental and endogenous origin [22], we enrolled children aged $>2$ years, characterized by a relatively stable adult-like intestinal microbial community [23]. In particular, the faecal microbiota of 19 atopic children and 12 healthy controls aged 4-14 years was characterized by means of the previously developed phylogenetic microarray platform High Taxonomic Fingerprint (HTF)Microbi.Array [24] and quantitative PCR (qPCR). Integrated of an additional probe pair for Akkermansia muciniphila, the HTF-Microbi.Array platform detects up to 31 intestinal bacterial groups and covers up to $95 \%$ of the human intestinal microbiota [25]. For our study faeces were selected since they represent the only realistic and reliable sample for a non-invasive study of the human intestinal microbiota.

\section{Methods}

\section{Subjects enrolled and study groups}

We enrolled 19 children (referred as atopics throughout the paper) with clinical diagnosis of allergy (rhinitis, asthma, grass pollen sensitization, allergic atopic dermatitis, oral allergy syndrome, cow's milk allergy) and encountering all the following criteria: (i) delivered naturally at term, (ii) breast fed for at least 3 months, (iii) aged between 4 and 14 years, (iv) no acute diseases for at least 2 weeks, (v) no antibiotic treatment in the last 3 months. In particular, 17 children presented allergic rhinitis, in 4 cases associated with asthma. Atopic dermatitis was observed in 8 cases of which 6 associated with rhinitis and inhalant sensitization and 1 with food allergy (Table 1). During the visit the children underwent a clinical evaluation and skin prick test for main food or inhalant allergens. Total and specific IgE determination was performed when clinically necessary. Fresh stool samples were collected within 3 days. As controls, 12 non-allergic children who encountered the same criteria above described but without family history of atopy were enrolled. All the children were routinely followed by the Paediatric Oncology and Haematology Unit Lalla Seràgnoli, Sant'Orsola-Malpighi Hospital, University of Bologna. Parents provided a written informed consent. Approval by the Ethics Committee of the Sant'Orsola-Malpighi Hospital was not needed for this study.

\section{Allergometric tests}

Skin prick tests were performed following established guidelines [26]. The following allergens were tested: cow's milk, egg, soy bean, wheat, peanut, codfish, grass pollen, Dermatophagoides pteronyssinus, Dermatophagoides farinae, and cat dander. Other allergens were tested on the basis of the child's history. Data of the skin prick tests were used to determine the presence of atopic sensitization in the definition of allergic or non-allergic atopic dermatitis. The determination of total serum IgE was performed by ELISA test; the values were assumed as normal or increased in comparison with the ones from children of the same age group [27]. The determination of specific $\operatorname{IgE}$ was 
Table 1 Allergic profile of the 19 atopic children enrolled in the study

\begin{tabular}{|c|c|c|c|c|c|c|}
\hline \multirow[b]{2}{*}{ Sample ID } & \multicolumn{6}{|c|}{ Clinical diagnosis of allergy } \\
\hline & $\mathrm{RC}^{\mathrm{a}}$ & $A^{b}$ & GPS $^{\mathrm{c}}$ & $A D^{d}$ & $O A S^{e}$ & $\mathrm{CMA}^{\mathrm{f}}$ \\
\hline A1 & $\sqrt{ }$ & & $\sqrt{ }$ & $\sqrt{ }$ & $\sqrt{ }$ & \\
\hline A2 & $\sqrt{ }$ & & $\sqrt{ }$ & $\sqrt{ }$ & & \\
\hline A3 & $\sqrt{ }$ & & $\sqrt{ }$ & & & \\
\hline A4 & $\sqrt{ }$ & $\sqrt{ }$ & $\sqrt{ }$ & & & \\
\hline A5 & $\sqrt{ }$ & $\sqrt{ }$ & $\sqrt{ }$ & & & \\
\hline A6 & $\sqrt{ }$ & & $\sqrt{ }$ & & $\sqrt{ }$ & $\sqrt{ }$ \\
\hline A7 & $\sqrt{ }$ & & $\sqrt{ }$ & $\sqrt{ }$ & & \\
\hline A8 & $\sqrt{ }$ & & $\sqrt{ }$ & $\sqrt{ }$ & & \\
\hline A9 & $\sqrt{ }$ & $\sqrt{ }$ & $\sqrt{ }$ & & $\sqrt{ }$ & $\sqrt{ }$ \\
\hline A10 & $\sqrt{ }$ & $\sqrt{ }$ & $\sqrt{ }$ & & & \\
\hline A12 & $\sqrt{ }$ & & $\sqrt{ }$ & $\sqrt{ }$ & & \\
\hline A13 & $\sqrt{ }$ & & $\sqrt{ }$ & $\sqrt{ }$ & & \\
\hline A14 & $\sqrt{ }$ & & $\sqrt{ }$ & & & \\
\hline A15 & & & & $\sqrt{ }$ & & \\
\hline A17 & $\sqrt{ }$ & & $\sqrt{ }$ & & & \\
\hline A19 & $\sqrt{ }$ & & $\sqrt{ }$ & & & \\
\hline A20 & & $\sqrt{ }$ & $\sqrt{ }$ & & & \\
\hline A21 & $\sqrt{ }$ & & & $\sqrt{ }$ & & \\
\hline A22 & $\sqrt{ }$ & & $\sqrt{ }$ & & & \\
\hline
\end{tabular}

${ }^{\mathrm{a}}$ Rhinitis.

${ }^{\mathrm{C}}$ Grass Pollen Sensitization.

${ }^{\mathrm{d}}$ Allergic Atopic Dermatitis.

eOral Allergy Syndrome.

fCow's Milk Allergy.

performed by UNICAP 1000 (Phadia) in all patients for the following allergens: cow's milk, egg, soy bean, wheat, peanut, codfish, Bermuda grass, timothy grass, $D$. pteronyssinus, $D$. farinae, and cat dander. Other allergens were tested on the basis of the child's history.

\section{DNA extraction and polymerase chain reaction (PCR)}

Total DNA from faecal material was extracted using QIAamp DNA Stool Mini Kit (Qiagen) according to the modified protocol reported by Candela et al. [24]. Final DNA concentration was determined using NanoDrop ND-1000 (NanoDrop Technologies). PCR amplifications were performed with Biometra Thermal Cycler $\mathrm{T}$ Gradient (Biometra). The $16 \mathrm{~S}$ rRNA gene was amplified using universal forward primer $27 \mathrm{~F}$ and reverse primer r1492, following the protocol described by Candela et al. [24]. PCR products were purified by using the Wizard SV gel and PCR clean-up System kit (Promega), eluted in $20 \mu \mathrm{l}$ of sterile water and quantified with the DNA 7500 LabChip Assay kit and BioAnalyzer 2100 (Agilent Technologies). All the oligonucleotide primers used for PCR reactions and probe pairs employed for the array construction were synthesized by Thermo Electron.

\section{HTF-microbi.Array analysis}

The HTF-Microbi.Array utilized in this study is based on the Ligase Detection Reaction-Universal Array (LDR-UA) approach [28] and enables specific detection and quantification of the $16 \mathrm{~S}$ rRNA from 31 phylogenetically related groups of the human intestinal microbiota (Additional file 1). The original HTF-Microbiarray [24] was updated to include a probe for the detection of $A$. muciniphila. The new probe was designed and validated as reported by Candela et al. [24] (Additional file 2). Sequences of the entire probe set of the HTF-Microbi.Array are reported in Additional file 3. Slide chemical treatment, array production, LDR protocol and hybridization conditions were carried out as previously reported $[28,29]$ with probe annealing set at $60^{\circ} \mathrm{C}$. The LDRs were carried out in a final volume of $20 \mu \mathrm{l}$ with $50 \mathrm{fmol}$ of PCR product. Two hundred and fifty fmol of synthetic template (5'-AGCCGCGAACACCACGATCGACCGGCGCGCGCAGCTGCAGCTTGCT CATG-3) were used for normalization purposes. All HTFMicrobi.Array experiments were performed in independent duplicates.

\section{Data analysis}

All arrays were scanned and processed according to the protocol and parameters already described by Candela et al. [24]. Fluorescence intensities (IF) were normalized on the basis of the synthetic ligation control signal: (a) outlier values (2.5-fold above or below the average) were discarded; (b) a correction factor was calculated in order to set the average IF of the ligation control to 50000 $(\mathrm{n}=6)$; (c) the correction factor was applied to both the probes and background IF values. Reproducibility of the experiments was assessed by calculating Pearson's correlation of the fluorescence signals between the two replicates. LDR experiments showing a Pearson's correlation coefficient $<0.95$ were repeated. Mean data from two replicated experiments were obtained and utilized for principal component analysis (PCA), box plot analysis and calculation of the probe relative IF contribution. Non-parametric Kruskal-Wallis test was used to determine whether the contribution of each bacterial group was significantly different between atopics and controls. Two-sided $t$-test was applied to evaluate whether the relative percentage contribution of each bacterial group was significantly different between the two groups. Correlation between variables was computed by Spearman rank correlation coefficient. Statistical analyses were performed by using Canoco package for Windows [30] and the R statistical software (www.r-project.org). 


\section{Quantitative PCR}

qPCR was carried out in a LightCycler instrument (Roche). Quantification of the $16 \mathrm{~S}$ rRNA gene of A. muciniphila, Faecalibacterium prausnitzii, Enterobacteriaceae, Clostridium cluster IV, Bifidobacterium and Lactobacillus group was performed with the genus-, group- or speciesspecific primers reported in Table 2. SYBR Green I fluorophore was used to correlate the amount of PCR product with the fluorescent signal. For quantification, standard curves were generated with known amounts of pCR2.1 (Invitrogen)-cloned $16 \mathrm{~S}$ rRNA gene from $A$. muciniphila (DSM22959), F. prausnitzii (DSM17677), E. coli (ATCC11105), Clostridium leptum (DSM753), Bifidobacterium animalis subsp. lactis (BI-07) and Lactobacillus acidophilus (LA-14). Amplification was carried out in a $20 \mu \mathrm{l}$ final volume containing $100 \mathrm{ng}$ of faecal DNA, $0.5 \mu \mathrm{M}$ of each primer and $4 \mu \mathrm{l}$ of LightCycler-FastStart DNA Master SYBR Green I (Roche). Amplifications were done under the following conditions: (i) starting preincubation at $95^{\circ} \mathrm{C}$ for $10 \mathrm{~min}$; (ii) amplification including 35 cycles of 4 steps each at the temperature transition rate of $20^{\circ} \mathrm{C} / \mathrm{s}$ : denaturation at $95^{\circ} \mathrm{C}$ for $15 \mathrm{~s}$, annealing at the appropriate temperature (Table 2) for $20 \mathrm{~s}$, extension at $72^{\circ} \mathrm{C}$ for $30 \mathrm{~s}$, and fluorescence acquisition at the appropriate temperature (Table 2) for $5 \mathrm{~s}$; (iii) melting curve analysis.

\section{Results}

Faecal microbiota profile of atopic children and healthy controls

The faecal microbiota of 19 atopic children and 12 healthy controls living in Italy was characterized by means of the HTF-Microbi.Array platform (Additional files 4 and 5) [24]. Hybridization experiments were performed in two replicates. Pearson's correlation coefficients ranging from 0.95 and 0.99 were achieved between the two replicates, proving the high reproducibility of the phylogenetic profiles obtained by the HTFMicrobi.Array platform. A PCA of the fluorescence signals from atopics and controls was carried out. The diagnosis of atopy was considered as a dummy environmental variable. As shown in Figure 1A, the principal components $\mathrm{PC} 2$ and $\mathrm{PC} 3$, which collectively represented only a minor fraction of the total variance $(9.7 \%)$, resulted in the separation of samples according to the health status. In order to identify the bacterial lineages showing differences in abundance between atopics and controls, probe fluorescence signals obtained from the HTF-Microbi.Array in atopics and controls were compared by box plot analysis (Additional file 6). Probes showing $P<0.3$ are represented in Figure 1B. Atopic children showed a tendency towards reduction of $A$. muciniphila, F. prausnitzii et rel. and Ruminococcus bromii et rel. (Clostridium cluster IV), and Clostridium cluster XIVa, and were enriched in Enterobacteriaceae, Bacillus clausii and Veillonella parvula.

On the basis of the HTF-Microbi.Array fluorescence data, the relative contribution of the major phyla in atopics and controls was calculated (Figure 2). At high taxonomic level, atopics and controls showed a comparable overall phylogenetic composition of the faecal microbiota. Indeed, their microbiota resulted largely dominated by Bacteroidetes and Firmicutes, which

Table 2 Primer sets used for the $16 \mathrm{~S}$ rRNA gene quantification of A. muciniphila, F. prausnitzii, Enterobacteriaceae, Clostridium cluster IV, Bifidobacterium and Lactobacillus group by qPCR. Amplicon size, annealing and fluorescence acquisition temperature are also reported

\begin{tabular}{|c|c|c|c|c|c|c|}
\hline $\begin{array}{c}\text { Target } \\
\text { microorganism }\end{array}$ & Primer set & Sequence (5' to $\left.3^{\prime}\right)$ & $\begin{array}{l}\text { Product } \\
\text { size (bp) }\end{array}$ & $\begin{array}{l}\text { Annealing } \\
\text { temp }\left({ }^{\circ} \mathrm{C}\right)\end{array}$ & $\begin{array}{l}\text { Fluorescence } \\
\text { acquisition } \\
\text { temp } \\
\left({ }^{\circ} \mathrm{C}\right)\end{array}$ & Reference \\
\hline \multirow[t]{2}{*}{ Akkermansia muciniphila } & AM1 & CAGCACGTGAAGGTGGGGAC & 349 & 63 & 88 & [31] \\
\hline & AM2 & CCTTGCGGTTGGCTTCAGAT & & & & \\
\hline \multirow{2}{*}{$\begin{array}{c}\text { Faecalibacterium } \\
\text { prausnitzii }\end{array}$} & Fprau223F & GATGGCCTCGCGTCCGATTAG & 199 & 67 & 85 & {$[32]$} \\
\hline & Fprau420R & CCGAAGACCTTCTTCCTCC & & & & \\
\hline \multirow[t]{2}{*}{ Enterobacteriaceae } & Eco1457F & CATTGACGTTACCCGCAGAAGAAG & 195 & 63 & 87 & {$[32]$} \\
\hline & Eco1652R & CTCTACGAGACTCAAGCTTGC & & & & \\
\hline \multirow[t]{2}{*}{ Clostridium Cl_IV } & S-*-Clos-0561-a-S-17 & TTACTGGGTGTAAAGGG & 588 & 60 & 85 & [33] \\
\hline & $\begin{array}{c}\text { S-*-Clept-1129.a-A- } \\
17\end{array}$ & TAGAGTGCTCTTGCGTA & & & & \\
\hline \multirow[t]{2}{*}{ Bifidobacterium } & bif-164 & GGGTGGTAATGCCGGATG & 523 & 60 & 90 & [34] \\
\hline & bif-662 & CCACCGTTACACCGGGAA & & & & \\
\hline \multirow[t]{2}{*}{ Lactobacillus group } & Lac1 & AGCAGTAGGGAATCTTCCA & 327 & 61 & 85 & [35] \\
\hline & Lac2 & ATTYCACCGCTACACATG & & & & \\
\hline
\end{tabular}




\section{A}

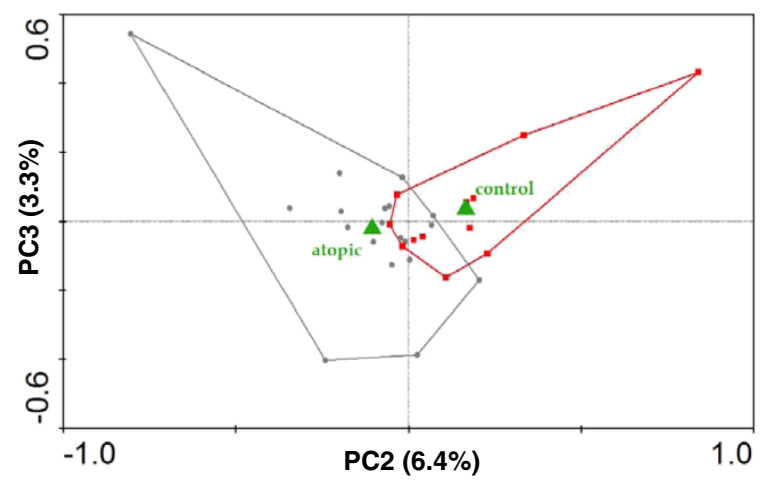

B
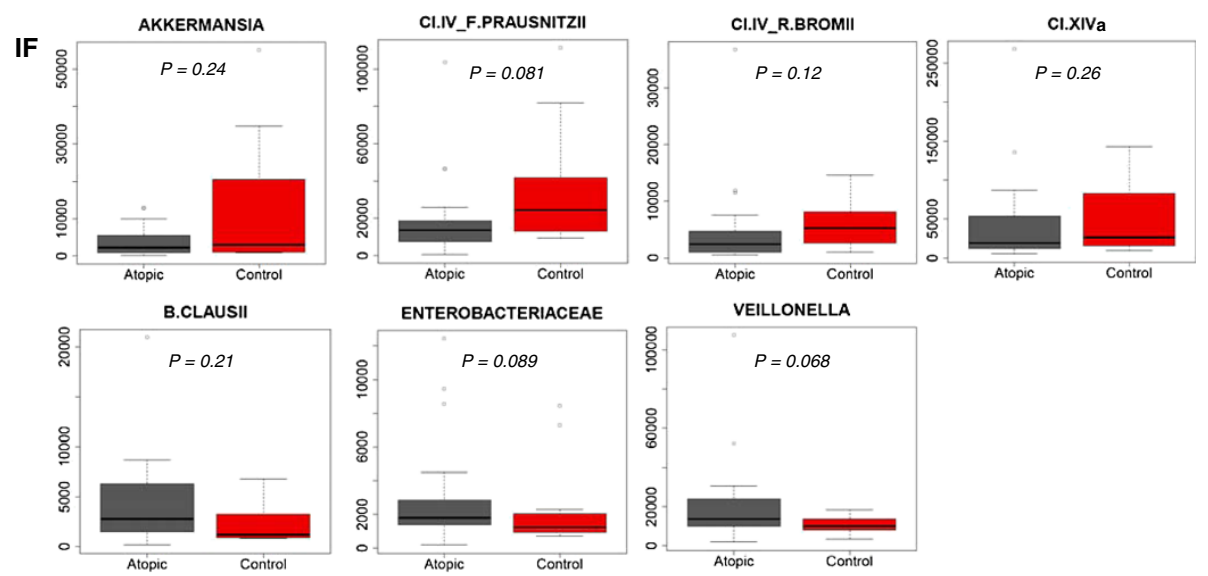

Figure 1 Analysis of the HTF-Microbi.Array fluorescence signals. A: PCA of the HTF-Microbi.Array fluorescence signals. Atopy or health status were considered as dummy environmental variables (green triangles) and indicated as atopic and control, respectively. Atopic subjects and healthy controls are indicated by gray circles and red squares, respectively. Second and third ordination axes are plotted showing 6.4\% and 3.3\% of the total variability in the dataset, respectively. B: Comparison of the HTF-Microbi.Array probe fluorescence signals between atopics and controls. Only probes showing a different trend between the two groups $(P<0.3)$ are shown.

together accounted for up to $90 \%$ of the faecal microbial community. With a relative abundance ranging from 1 to 5\%, Fusobacteria, Actinobacteria and Proteobacteria were sub-dominant components. However, focusing at lower taxonomic level, significant differences in the relative contribution of certain microbial groups were detected. In particular, atopics were characterized by a lower relative contribution of members of the Clostridium cluster IV (atopics, 20.9\% - controls, 28.7\%; $P=0.01)$ and a concomitant relative increase in Enterobacteriaceae (atopics, 2.4\% - controls, 1.2\%; $P=0.009$ ) and Fusobacteria (atopics, 1.9\% - controls, 1.2\%; $P=0.001$.

The abundance of $F$. prausnitzii, A. muciniphila, Enterobacteriaceae, Clostridium cluster IV, Bifidobacterium and Lactobacillus group in the faecal microbiota of atopics and controls was investigated by qPCR analysis of the $16 \mathrm{~S}$ rRNA gene. As reported in Table 3, respect to healthy controls, atopics were significantly depleted in F. prausnitzii, A. muciniphila and members of the Clostridium cluster $I V$, and tended to be depleted in Bifidobacterium and enriched in Enterobacteriaceae.

\section{Correlation among faecal microbiota, diagnosis of allergy and total $\lg \mathrm{E}$}

In order to investigate whether the profile of the faecal microbial community of atopics correlated with their allergy profile (Table 1), a PCA of the HTF-Microbi.Array fluorescence signals from atopics was carried out. PCA was performed by considering the types of allergic response as dummy environmental variables. No separation of the atopic children according to the specific diagnosis of rhinitis, asthma, grass pollen sensitization, allergic atopic dermatitis, oral allergy syndrome and cow's milk allergy was obtained, proving that the atopy- 


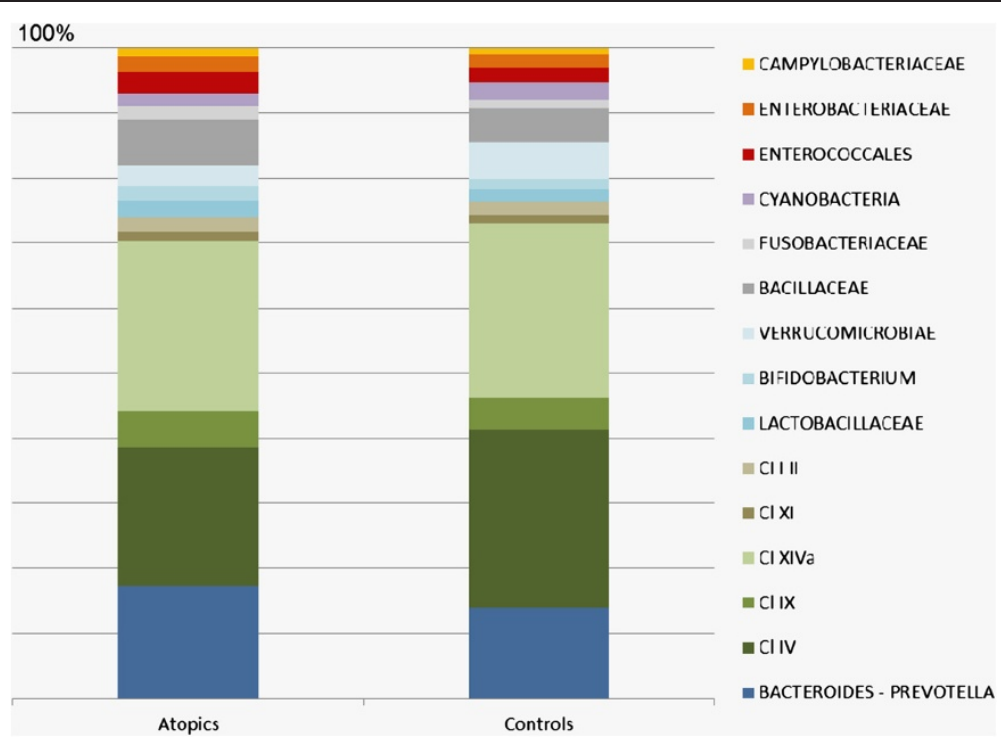

Figure 2 Relative contribution of the principal intestinal microbial groups in the faecal microbiota of atopics and controls. For each HTF-Microbi.Array probe, the relative fluorescence contribution was calculated as percentage of the total fluorescence. Sub-probes were excluded. Data represent the mean of the probe relative fluorescence contribution in atopics $(n=19)$ and controls $(n=12)$. $P$ values derive from a two-sided t-test.

related dysbioses of the faecal microbiota are independent of the specific atopic outcome (data not shown).

In a subset of 10 atopy cases with clinical relevance the total serum IgE levels were determined. Total IgE ranged from 138 to $855 \mathrm{ku} / \mathrm{L}$ (geometric mean: $326 \mathrm{ku} /$ L), a value above the normal for age [27]. In order to investigate whether in this subset of 10 atopics IgE correlated with the relative abundance of a specific microbial group in the faeces, Spearman rank correlation coefficients between the probe relative fluorescence signals and the IgE levels were calculated. According to our data no significant correlation was determined. However, a tendency towards an inverse correlation with IgE was obtained for $L$. casei et rel. $(\rho=0.52 ; P=0.100)$, while Clostridium cluster IX abundance tended to be positively correlated with total $\operatorname{IgE}(\rho=0.60 ; P=0.073)$ (Figure 3).

\section{Discussion}

In the present paper we combined two culture-independent molecular approaches, HTF-Microbi.Array and qPCR, for a pilot characterization of the atopy-associated dysbiosis of the intestinal microbiota in 19 atopic children living in Italy. At high phylogenetic level both atopics and controls showed a comparable overall microbiota profile where Firmicutes and Bacteroidetes constituted the two dominant divisions. However, focusing at lower taxonomic level, the intestinal microbiota of atopic children was characterized by a significant depletion in members of the Clostridium cluster IV, F. prausnitzii, A. muciniphila and a corresponding increase of the relative abundance of Enterobacteriaceae.
In a case-control DGGE-based study of the faecal microbiota from 20 allergic and 20 non-allergic 5-year-old Estonian children, Stsepetova et al. [36] reported a less diverse composition in the faecal microbiota from atopic children but, according to the Authors, no bacterial targets could distinguish infants with or without atopy. However, the DGGE-based approach allowed to consider only the dominant fraction of the intestinal microbiota, remaining blind with respect to the whole phylogenetic complexity of the ecosystem. In an elegant $16 \mathrm{~S}$ rDNA pyrosequencing-based dynamic study, Hong et al. [37] addressed the differences in the microbiota succession between 3 infants with and 4 without eczema over four time points until 24 months of age. Even if age was shown to be the dominant factor mediating microbiota changes, matched by age eczema infants were characterized by a higher abundance of the enterobacteria Klebsiella and Shigella as well as Enterococcus, while Bifidobacterium showed a higher abundance in non-eczema ones. These last data are in general agreement with the intestinal microbiota dysbioses observed in our study.

Although Bifidobacterium and Lactobacillus have been traditionally indicated as possible protective factors against atopic disease in childhood [16], we did not detect any significant differences in these health-promoting genera between atopics and controls, confirming previous findings reported by Penders et al. $[3,18]$. However, molecular studies at the species level showed a different distribution of the Bifidobacterium and Lactobacillus species between allergic and non-allergic children [36,38], suggesting a potential species-specific effect of 
Table 3 qPCR quantification of $F$. prausnitzii, $A$. muciniphila, Enterobacteriaceae, Clostridium cluster IV, Bifidobacterium and Lactobacillus group in the faecal microbiota of atopics and healthy controls

\begin{tabular}{cccc}
\hline & \multicolumn{3}{c}{ 16S rRNA gene copies/ $\boldsymbol{\mu g}$ fecal } \\
\hline DNA & \\
\hline Facterial species/group & Atopics & Controls & $\boldsymbol{P}$ value \\
Akkermansia muciniphila & $3.01 \mathrm{E}+05$ & $5.03 \mathrm{E}+05$ & $\mathbf{0 . 0 1 9 0}$ \\
Enterobacteriaceae & $3.86 \mathrm{E}+04$ & $1.19 \mathrm{E}+04$ & 0.3500 \\
Clostridium cluster IV & $4.46 \mathrm{E}+06$ & $1.55 \mathrm{E}+07$ & $\mathbf{0 . 0 0 3 5}$ \\
Bifidobacterium & $1.08 \mathrm{E}+06$ & $1.72 \mathrm{E}+06$ & 0.0850 \\
Lactobacillus group & $3.75 \mathrm{E}+02$ & $5.48 \mathrm{E}+02$ & 0.6410 \\
\hline
\end{tabular}

For each bacterial species/group, the mean $16 \mathrm{~S}$ rRNA copy number per $\mu \mathrm{g}$ of faecal DNA is reported.

Bifidobacterium and Lactobacillus in the etiology of atopic disorders.

The atopy-related microbiota dysbioses we depicted in our cohort of 19 children were independent of their peculiar allergic profile. A subset of 10 atopics underwent clinical evaluation of total IgE level and the correlation between $\operatorname{IgE}$ and the relative abundance of specific microbial groups in the faeces was explored. Even if no significant correlation was determined, L. casei et rel. and Clostridium cluster IX tended to be negatively and positively correlated with IgE, respectively. Interestingly, Ogawa et al. [39] demonstrated that orally administered L. casei was effective in the control of the IgE levels in human allergic reactions and, recently, Schiffer et al. [40] reported that L. casei could inhibit the effector phase of immune inflammation in vivo. Finally, Penders et al. [38] showed a decreased risk of atopic dermatitis in children colonized by L. paracasei, a member of the $L$. casei et rel. group. Even if these studies may support the tendency towards inverse correlation between $L$. casei et rel. and IgE level we observed in our study, caution must be taken in considering these data since only a low number of children were analyzed.

Characterized by a decrease of the absolute levels of Clostridium cluster IV, F. prausnitzii and A. muciniphila, as well as a corresponding increase in the relative abundance of Enterobacteriaceae, the atopy-associated intestinal microbial community we described in this study is depleted in key immunomodulatory members of the human intestinal microbiota and possibly enriched in pro-inflammatory "pathobionts" [41]. By the specific induction of $\mathrm{T}$ regs, members of the Clostridium cluster IV have been demonstrated to be strategic for maintaining the immune homeostasis [42]. Analogously, providing a vast range of antiinflammatory effects, F. prausnitzii has been considered as a crucial microorganism for gut homeostasis [43]. Finally, A. muciniphila is a common member of the human intestinal tract which has been recently associated with a protective/anti-inflammatory role in healthy gut [44]. On the other hand, Enterobacteriaceae have been reported to prosper in the context of a host-mediated inflammatory response [45]. Capable to venture more deeply in the mucus layer and establish a close interaction with the epithelial surface, members of Enterobacteriaceae concur in the induction of a pro-inflammatory response and further consolidate the host inflammatory status. Thus, similarly to the one characterized in IBD [43,46-48], the atopy-associated microbiota can represent an inflammogenic microbial consortium which can contribute to the severity of the disease [7].

\section{Conclusion}

Atopic children were depleted in specific members of the intestinal microbiota that, capable to orchestrate a broad spectrum of inflammatory and regulatory $\mathrm{T}$ cell responses, have been reported as fundamental for the immune homeostasis. The decrease of these key immunomodulatory symbionts in the gastrointestinal tract - as well as the
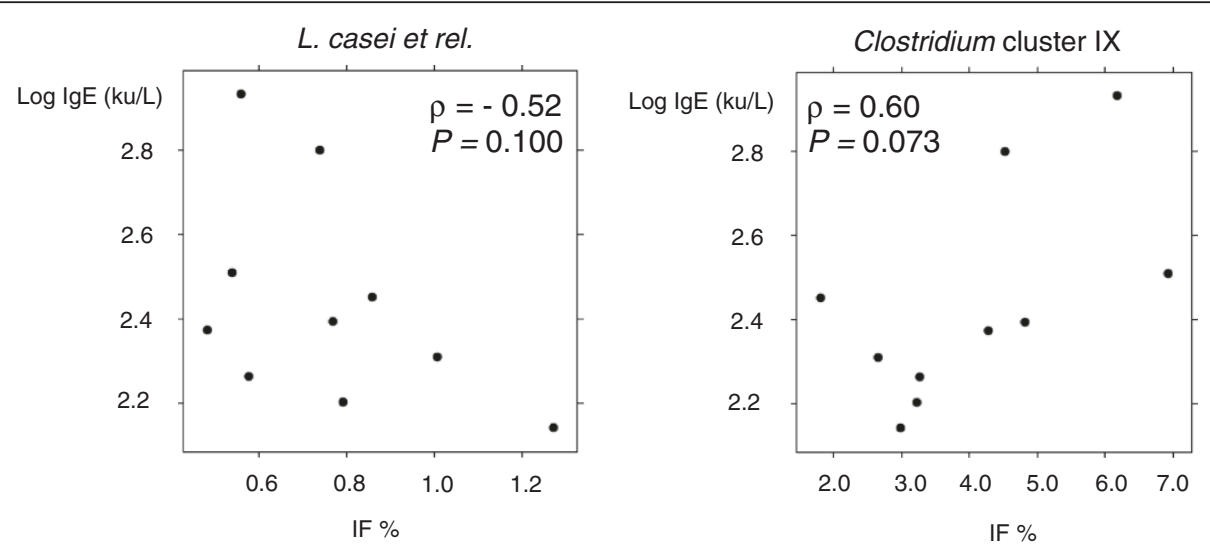

Figure 3 Spearman rank correlation between total IgE level and the abundance of $L$. casei et rel. and Clostridium cluster IX in the stools from a subset of 10 atopic children. 
corresponding increase in relative abundance of pro-inflammatory Enterobacteriaceae - support the immune deregulation and, in the context of an atopic host, can sustain an inflammatory status throughout the body. Since the atopy-related dysbioses of the intestinal microbiota can contribute to the severity of the disease, atopy treatment may be facilitated by redressing these microbiological unbalances. To this aim, advantages can be taken from the possibility to manipulate the microbiota plasticity with diet or pharmaceutical prebiotics and probiotics. However, the phylogenetic resolution of the data reported in our study needs to be implemented by deep $16 \mathrm{~S}$ rDNA sequencing. Moreover, metatranscriptomic studies can be carried out. Linking the phylogenetic structure of the intestinal microbiota with its specific functional activities, the metatranscriptomic characterization of the intestinal microbiota in atopic children could reveal the possible pathogenic mechanisms behind the atopy-related microbiota dysbioses.

\section{Additional files}

\section{Additional file 1: Phylogenetically related groups target of the HTF-} Microbi.Array.

Additional file 2: Probe specificity tests for Akkermansia muciniphila. Data refer to independent duplicates obtained using $50 \mathrm{fmol}$ of purified $16 \mathrm{~S}$ rRNA PCR product. $X$ axis shows the ZipCode for each probe pair; in both figures, "1B" represents the ZipCode associated to A. muciniphila. Y axis shows the average fluorescence intensities (IF) for each probe pair. Fluorescence between the two replicates was not normalized. Blue stars over the fluorescence bars indicate the probes that gave a positive response with $P<0.01$. Red dots indicate that one or two replicates out of four for each ZipCode were excluded because of having an IF 2.5-fold above or below the average of the spots.

Additional file 3: HTF-Microbi.Array probe list. Sequences $\left(5^{\prime}->3^{\prime}\right)$ for both discriminating (DS) and common probe (CP) are reported, as well as major thermodynamic parameters [melting temperature (Tm), length (bp), number of degenerated bases (Deg)].

Additional file 4: HTF-Microbi.Array raw fluorescence data obtained from the analysis of faecal stools from 19 atopic children (A) and 12 healthy controls (C).

Additional file 5: Layout of the HTF-Microbi.Array and complete ZipCode sequences.

Additional file 6: Box plots of the HTF-Microbi.Array fluorescence signals from atopics and controls. $P$ values corresponding to the difference in fluorescence response between the two groups are indicated for each probe.

\section{Abbreviations}

A: Asthma; AD: Allergic Atopic Dermatitis; CMA: Cow's Milk Allergy; GPS: Grass Pollen Sensitization; HTF-Microbi.Array: High Taxonomic Fingerprint-Microbi.Array; IBD: Inflammatory Bowel Diseases; IF: Fluorescence Intensities; KOALA: Is (in Dutch) an acronym for Child Parent and health, Lifestyle and Genetic constitution; LDR-UA: Ligase Detection ReactionUniversal Array; OAS: Oral Allergy Syndrome; PCA: Principal Component Analysis; PC1 2 and 3: Principal Components 1, 2 and 3; RC: Rhinitis.

\section{Acknowledgments}

This work was funded by the Micro(bi)array project of the University of Bologna, Italy. Our thanks to Giada Caredda for the support in experimental phase.

\section{Author details}

'Department of Pharmaceutical Sciences, University of Bologna, Via Belmeloro 6, Bologna 40126, Italy. ${ }^{2}$ Institute of Biomedical Technologies, Italian National Research Council, Milan, Italy. ${ }^{3}$ Paediatric Oncology and Haematology Unit Lalla Seràgnoli, Sant'Orsola-Malpighi Hospital, University of Bologna, Bologna, Italy. ${ }^{4}$ Department of Gynecological, Obstetrical and Pediatric Sciences, Sant'Orsola-Malpighi Hospital, University of Bologna, Bologna, Italy.

\section{Authors' contributions}

MC conceived and designed the experiments, analyzed the data and wrote the first draft of the paper. SR and ST performed faecal microbial DNA extraction, $16 \mathrm{~S}$ rDNA amplification and purification, GPCR bacterial quantifications and PCA analysis. MS, CC, GDB performed all the HTF-Microbi. Array hybridization experiments and data analysis. RM, GR and AP enrolled subjects and performed skin prick test and IgE determination. PB conceived and designed the experiments. All authors read and approved the final manuscript.

Received: 27 September 2011 Accepted: 6 June 2012

Published: 6 June 2012

\section{References}

1. Romagnani S: Regulatory T cells: which role in the pathogenesis and treatment of allergic disorders? Allergy 2006, 61:3-14.

2. Ngoc PL, Gold DR, Tzianabos AO, Weiss ST, Celedón JC: Cytokines, allergy, and asthma. Curr Opin Allergy Clin Immunol 2005, 5:161-166.

3. Penders J, Stobberingh EE, van den Brandt PA, Thijs C: The role of the intestinal microbiota in the development of atopic disorders. Allergy 2007, 62:1223-1236.

4. Ehlers S, Kaufmann SH, Participants of the 99(th) Dahlem Conference: Infection, inflammation, and chronic diseases: consequences of a modern lifestyle. Trends Immunol 2010, 31:184-190.

5. Rautava S, Ruuskanen O, Ouwehand A, Salminen S, Isolauri E: The hygiene hypothesis of atopic disease-an extended version. J Pediatr Gastroenterol Nutr 2004, 38:378-388.

6. De Filippo C, Cavalieri D, Di Paola M, Ramazzotti M, Poullet JB, Massart S, Collini S, Pieraccini G, Lionetti P: Impact of diet in shaping gut microbiota revealed by a comparative study in children from Europe and rural Africa. Proc Natl Acad Sci U S A 2010, 107:14691-14696.

7. Kau AL, Ahern PP, Griffin NW, Goodman AL, Gordon Jl: Human nutrition, the gut microbiome and the immune system. Nature 2011, 474:327-336.

8. Lee YK, Mazmanian SK: Has the microbiota played a critical role in the evolution of the adaptive immune system? Science 2010, 330:1768-1773.

9. Egert M, de Graaf AA, Smidt H, de Vos WM, Venema K: Beyond diversity: functional microbiomics of the human colon. Trends Microbiol 2006, 14:86-91.

10. Mazmanian SK, Round JL, Kasper DL: A microbial symbiosis factor prevents intestinal inflammatory disease. Nature 2008, 453:620-625.

11. Gaboriau-Routhiau V, Rakotobe S, Lécuyer E, Mulder I, Lan A, Bridonneau C, Rochet V, Pisi A, De Paepe M, Brandi G, Eberl G, Snel J, Kelly D, CerfBensussan N: The key role of segmented filamentous bacteria in the coordinated maturation of gut helper T cell responses. Immunity 2009, 31:677-689.

12. Neish AS: Microbes in gastrointestinal health and disease. Gastroenterology 2009, 136:65-80.

13. Maslowski KM, Mackay CR: Diet, gut microbiota and immune responses. Nat Immunol 2011, 12:5-9.

14. Penders J, Thijs C, van den Brandt PA, Kummeling I, Snijders B, Stelma F, Adams $H$, van Ree R, Stobberingh EE: Gut microbiota composition and development of atopic manifestations in infancy: the KOALA Birth Cohort Study. Gut 2007, 56:661-667.

15. Kalliomäki M, Kirjavainen P, Eerola E, Kero P, Salminen S, Isolauri E: Distinct patterns of neonatal gut microflora in infants in whom atopy was and was not developing. J Allergy Clin Immunol 2001, 107:129-134.

16. Vael C, Desager K: The importance of the development of the intestinal microbiota in infancy. Curr Opin Pediatr 2009, 21:794-800.

17. Murray CS, Tannock GW, Simon MA, Harmsen HJ, Welling GW, Custovic A, Woodcock A: Fecal microbiota in sensitized wheezy and non-sensitized non-wheezy children: a nested case-control study. Clin Exp Allergy 2005, 35:741-745 
18. Penders J, Stobberingh EE, Thijs C, Adams H, Vink C, van Ree R, van den Brandt PA: Molecular fingerprinting of the intestinal microbiota of infants in whom atopic eczema was or was not developing. Clin Exp Allergy 2006, 36:1602-1608

19. Bisgaard H, Li N, Bonnelykke K, Chawes BL, Skov T, Paludan-Müller G, Stokholm J, Smith B, Krogfelt KA: Reduced diversity of the intestinal microbiota during infancy is associated with increased risk of allergic disease at school age. J Allergy Clin Immunol 2011, 128:646-652.

20. Abrahamsson TR, Jakobsson HE, Andersson AF, Björkstén B, Engstrand L, Jenmalm MC: Low diversity of the gut microbiota in infants with atopic eczema. J Allergy Clin Immunol 2012, 129:434-440.

21. Blaser MJ, Falkow S: What are the consequences of the disappearing human microbiota? Nat Rev Microbiol 2009, 7:887-894.

22. Dominguez-Bello MG, Blaser MJ, Ley RE, Knight R: Development of the human gastrointestinal microbiota and insights from high-throughput sequencing. Gastroenterology 2011, 140:1713-1719.

23. Palmer C, Bik EM, DiGiulio DB, Relman DA, Brown PO: Development of the human infant intestinal microbiota. PLOS Biol 2007, 5:e177.

24. Candela M, Consolandi C, Severgnini M, Biagi E, Castiglioni B, Vitali B, De Bellis $G$, Brigidi P: High taxonomic level fingerprint of the human intestinal microbiota by ligase detection reaction-universal array approach. BMC Microbiol 2010, 19:116.

25. Rajilić-Stojanović M, Smidt H, de Vos WM: Diversity of the human gastrointestinal tract microbiota revisited. Environ Microbiol 2007, 9:2125-2136.

26. Dreborg S, Frew A: Position Paper EAACl: allergen standardization and skin tests. Allergy 1993, 48:49-82.

27. Cavagni G, Capra T, Suzzani L, Nori G, Derin FM, Dusi R, Bernasconi S: Valori di IgE in bambini sani, non atopici. Folia Allergol Immunol Clin 1980, 27:273.

28. Castiglioni B, Rizzi E, Frosini A, Sivonen K, Rajaniemi P, Rantala A, Mugnai MA, Ventura S, Wilmotte A, Boutte C, Grubisic S, Balthasart P, Consolandi C, Bordoni R, Mezzelani A, Battaglia C, De Bellis G: Development of a universal microarray based on the ligation detection reaction and $16 \mathrm{~S}$ rrna gene polymorphism to target diversity of cyanobacteria. Appl Environ Microbiol 2004, 70:7161-7172.

29. Consolandi C, Severgnini M, Castiglioni B, Bordoni R, Frosini A, Battaglia C, Rossi Bernardi L, De Bellis G: A structured chitosan-based platform for biomolecule attachment to solid surfaces: application to DNA microarray preparation. Bioconjug Chem 2006, 17:371-377.

30. Leps J, Smilauer P: Multivariate analysis of ecological data using CANOCO. Cambridge: Cambridge University Press; 2003.

31. Collado MC, Derrien M, Isolauri E, de Vos WM, Salminem S: Intestinal integrity and Akkermansia muciniphila, a mucin-degrading member of the intestinal microbiota present in infants, adults and the elderly. Appl Environ Microbiol 2007, 23:7767-7770.

32. Bartosch S, Fite A, Macfarlane GT, McMurdo MET: Characterization of bacterial communities in feces from healthy elderly volunteers and hospitalized elderly patients by using Real-Time PCR and effects of antibiotic treatment on the fecal microbiota. Appl Environ Microbiol 2004, 6:3575-3581.

33. Van Dyke MI, McCarthy AJ: Molecular biological detection and characterization of Clostridium populations in municipal landfill sites. Appl Environ Microbiol 2002, 4:2049-2053.

34. Kok RG, de Waal A, Schut F, Welling GW, Weenk G, Hellingwerf KJ: Specific detection and analysis of a probiotic Bifidobacterium strain in infant feces. Appl Environ Microbiol 1996, 62:3668-3672.

35. Walter J, Hertel C, Tannock GW, Lis CM, Munro K, Hammes WP: Detection of Lactobacillus, Pediococcus, Leuconostoc and Weissella species in human feces by using group-specific PCR primers and denaturing gradient gel electrophoresis. Appl Environ Microbiol 2001, 67:2578-2585.

36. Stsepetova J, Sepp E, Julge K, Vaughan E, Mikelsaar M, de Vos WM: Molecularly assessed shifts of Bifidobacterium ssp. and less diverse microbial communities are characteristic of 5-year-old allergic children. FEMS Immunol Med Microbiol 2007, 51:260-269.

37. Hong PY, Lee BW, Aw M, Shek LP, Yap GC, Chua KY, Liu WT: Comparative analysis of fecal microbiota in infants with and without eczema. PLoS One 2010, 5:e9964.

38. Penders J, Thijs C, Mommers M, Stobberingh EE, Dompeling E, Reijmerink $\mathrm{NE}$, van den Brandt PA, Kerkhof M, Koppelman GH, Postma DS: Intestinal lactobacilli and the DC-SIGN gene for their recognition by dendritic cells play a role in the aetiology of allergic manifestations. Microbiology 2010, 156:3298-3305.

39. Ogawa T, Hashikawa S, Asai Y, Sakamoto H, Yasuda K, Makimura Y: A new synbiotic, Lactobacillus casei subsp. casei together with dextran, reduces murine and human allergic reaction. FEMS Immunol Med Microbiol 2006, 46:400-409.

40. Schiffer C, Lalanne Al, Cassard L, Mancardi DA, Malbec O, Bruhns P, Dif F, Daëron M: A strain of Lactobacillus casei inhibits the effector phase of immune inflammation. J Immunol 2011, 187:2646-2655.

41. Chow J, Mazmanian SK: A pathobiont of the microbiota balances host colonization and intestinal inflammation. Cell Host Microbe 2010, 7:265-276.

42. Atarashi $K$, Tanoue T, Shima T, Imaoka A, Kuwahara T, Momose $Y$, Cheng G, Yamasaki S, Saito T, Ohba Y, Taniguchi T, Takeda K, Hori S, Ivanov II, Umesaki Y, Itoh K, Honda K: Induction of colonic regulatory T cells by indigenous Clostridium species. Science 2011, 331:337-341.

43. Sokol H, Pigneur B, Watterlot L, Lakhdari O, Bermúdez-Humarán LG, Gratadoux JJ, Blugeon S, Bridonneau C, Furet JP, Corthier G, Grangette C, Vasquez N, Pochart P, Trugnan G, Thomas G, Blottière HM, Doré J, Marteau P, Seksik P, Langella P: Faecalibacterium prausnitzii is an anti-inflammatory commensal bacterium identified by gut microbiota analysis of Crohn disease patients. Proc Natl Acad Sci U S A 2008, 105:16731-16736.

44. Png CW, Lindén SK, Gilshenan KS, Zoetendal EG, McSweeney CS, Sly LI, McGuckin MA, Florin TH: Mucolytic bacteria with increased prevalence in IBD mucosa augment in vitro utilization of mucin by other bacteria. Am J Gastroenterol 2010, 105:2420-2428.

45. Lupp C, Robertson ML, Wickham ME, Sekirov I, Champion OL, Gaynor EC, Finlay BB: Host-mediated inflammation disrupts the intestinal microbiota and promotes the overgrowth of Enterobacteriaceae. Cell Host Microbe 2007, 2:204.

46. Frank DN, St Amand AL, Feldman RA, Boedeker EC, Harpaz N, Pace NR: Molecular-phylogenetic characterization of microbial community imbalances in human inflammatory bowel diseases. Proc Natl Acad Sci U S A 2007, 104:13780-13785.

47. Sokol H, Seksik P, Furet JP, Firmesse O, Nion-Larmurier I, Beaugerie L, Cosnes J, Corthier G, Marteau P, Doré J: Low counts of Faecalibacterium prausnitzii in colitis microbiota. Inflamm Bowel Dis 2009, 15:1183-1189.

48. Schwiertz A, Jacobi M, Frick JS, Richter M, Rusch K, Köhler H: Microbiota in pediatric inflammatory bowel disease. J Pediatr 2010, 157:240-244.

doi:10.1186/1471-2180-12-95

Cite this article as: Candela et al:: Unbalance of intestinal microbiota in atopic children. BMC Microbiology 2012 12:95.

\section{Submit your next manuscript to BioMed Central and take full advantage of:}

- Convenient online submission

- Thorough peer review

- No space constraints or color figure charges

- Immediate publication on acceptance

- Inclusion in PubMed, CAS, Scopus and Google Scholar

- Research which is freely available for redistribution 